\title{
Asymmetry and Leverage in Realized Volatility
}

\author{
Manabu Asai \\ Faculty of Economics \\ Soka University, Japan \\ Michael McAleer \\ School of Economics and Commerce \\ University of Western Australia \\ and \\ Econometric Institute \\ Erasmus University Rotterdam
}

Marcelo C. Medeiros

Department of Economics

Pontifical Catholic University of Rio de Janeiro

\section{EI 2008-31}

Revised: November 2008

* The authors are grateful to Marcel Scharth for efficient research assistance. For financial support, the first author acknowledges the Japan Ministry of Education, Culture, Sports, Science and Technology, the Japan Society for the Promotion of Science, and the Australian Academy of Science, the second author is most grateful to the Australian Research Council, and the third author wishes to acknowledge CNPq, Brazil. 


\begin{abstract}
A wide variety of conditional and stochastic variance models has been used to estimate latent volatility (or risk). In both the conditional and stochastic volatility literature, there has been some confusion between the definitions of asymmetry and leverage. In this paper, we first show the relationship among conditional, stochastic, integrated and realized volatilities. Then we develop a new asymmetric volatility model, which takes account of small and large, and positive and negative, shocks. Using the new specification, we examine alternative volatility models that have recently been developed and estimated in order to understand the differences and similarities in the definitions of asymmetry and leverage. We extend the new specification to realized volatility by taking account of measurement errors. As an empirical example, we apply the new model to the realized volatility of Standard and Poor's 500 Composite Index using Efficient Importance Sampling to show the new specification of asymmetry significantly improves the goodness of fit.
\end{abstract}




\section{Introduction}

The accurate specification and modelling of risk are integral to optimal portfolio selection and risk management using high frequency and ultra high frequency data. In this context, a wide variety of conditional and stochastic variance models has been used to estimate latent volatility (or risk) using high frequency data, while the availability of tick data has led to alternative models of realized volatility to estimate integrated volatility in analysing ultra high frequency data (see McAleer (2005) for a comprehensive review of univariate and multivariate, and symmetric and asymmetric, conditional and stochastic volatility models, and Asai, McAleer and Yu (2006) for a detailed review of alternative specifications and estimation algorithms for multivariate stochastic volatility models).

In the framework of diffusion processes, the daily variance of stock return is expressed as an integral of the intraday variance, which is called the integrated variance. If the microstructure noise is ignored, we may estimate it by the sum of squared returns of ultra high frequency data. Such an estimator is called the realized variance, which corresponds to an estimate of the integrated variance, namely the true daily variance. In the paper, we refer to the square root of the realized variance as the Realized Volatility (RV). For a recent extensive review of the RV literature, see McAleer and Medeiros (2008).

Upon estimating RV by using ultra high frequency data, one of the major problems that arises is microstructure noise. Several authors have proposed alternative methods for removing the microstructure noise (see, for example, Bandi and Russell (2006), Barndorff-Nielsen, Hansen, Lunde and Shephard (2007), Zhang, Mykland and Aït-Sahalia (2005), and Hansen, Large and Lunde (2008)). Some methods provide bias-corrected and consistent estimators of the integrated variance, but other methods do not. Recently, Asai, McAleer and Medeiros (2008) have shown that, even when a bias-corrected and consistent estimator is used, non-negligible measurement errors remain in estimating and forecasting RV.

The purpose of the paper is to propose a new specification of the asymmetric RV model. Before doing so, we need to clarify three points: (i) the relationship among conditional, stochastic, integrated and realized volatility; (ii) the confusion between the definitions of asymmetry and leverage; and (iii) the way in which to estimate a model of integrated volatility using RV data. 
Regarding the second point, this paper proposes a general asymmetric volatility model. Based on the new specification, we examine alternative univariate volatility models that have recently been developed and estimated in order to understand the differences and similarities in the definitions of asymmetry and leverage. We focus on standardized and/or unstandardized leverage and size effects, and analyse five univariate SV models, namely the basic SV model, SV model with standardized leverage, and three different types of asymmetric SV models (see Harvey and Shephard (1996), Danielsson (1994), and Asai and McAleer (2005, 2008)). Then we evaluate the differential impacts of positive and negative shocks to returns of equal magnitude on future volatility, namely symmetry, asymmetry, type I asymmetry (or leverage), type II asymmetry, type III asymmetry and type IV asymmetry. The general model proposed here is classified as type IV asymmetry.

With respect to the third point, we propose a new asymmetric model for RV by extending the general asymmetric volatility model, with an additional term in order to capture RV errors. It should be noted that introducing a correction for measurement error in the RV process renders the true volatility process unobservable. In order to estimate the proposed model, we employ the efficient importance sampling (EIS) ML method proposed by Liesenfeld and Richard (2003, 2006). The EIS evaluates the log-likelihood function of the model, including the latent process, by using simulations, such as the Monte Carlo Likelihood (MCL) technique of Durbin and Koopman (1997). Compared with the MCL method, the EIS method is applicable to various kinds of latent models (see also the discussion in Liesenfeld and Richard (2003)).

The remainder of the paper is organized as follows. Section 2 shows the connection among conditional, stochastic, integrated and realized volatility. Section 3 develops a general asymmetric volatility model. We examine five kinds of asymmetric SV models, and propose classifications for symmetric and/or asymmetric effects. By using the structure of asymmetric effects, Section 4 proposes a new model for RV based on correcting for RV errors. Section 5 discusses the EIS-ML method, while Section 6 presents the empirical results for the RV model using Standard and Poor's 500 Composite Index. Section 7 gives some concluding remarks.

\section{Instantaneous, Integrated and Realized Volatility}


Let $p(t+\tau)$ is the logarithmic price of a given asset at time $\tau \quad(0 \leq \tau \leq 1)$ on day $t$ $(t=1,2, \ldots)$. We assume that $p(t+\tau)$ follows a continuous time diffusion process,

$$
d p(t+\tau)=\mu(t+\tau) d \tau+\sigma(t+\tau) d W(t+\tau)
$$

where $\mu(t+\tau)$ is the drift component, $\sigma(t+\tau)$ is the instantaneous volatility (or standard deviation), and $W(t+\tau)$ is a standard Brownian motion. Let $r_{t}$ be the daily returns, defined as $r_{t}=p(t)-p(t-1)$. Conditionally on

$$
\mathfrak{I}_{t} \equiv \mathfrak{I}\{\mu(t+\tau-1), \sigma(t+\tau-1)\}_{\tau=0}^{\tau=1},
$$

which is the $\sigma$-algebra (information set) generated by the sample paths of $\mu(t+\tau-1)$ and $\sigma(t+\tau-1) \quad(0 \leq \tau \leq 1)$, we have

$$
r_{t} \mid \mathfrak{I}_{t} \sim N\left(\int_{0}^{1} \mu(t+\tau-1) d \tau, \int_{0}^{1} \sigma^{2}(t+\tau-1) d \tau\right)
$$

The term $V_{t}^{2}=\int_{0}^{1} \sigma^{2}(t+\tau-1) d \tau$ is known as the integrated variance, which is a measure of the day-t ex post volatility. The integrated variance is typically the object of interest as a measure of the true daily volatility.

With respect to the model of the instantaneous volatility, there are several specifications, which are called "continuous-time Stochastic Volatility (SV)" models (see Ghysels, Harvey and Renault (1996), for example). Hull and White (1987) allow the squared-volatility to follow a diffusion process,

$$
d \sigma^{2}=\alpha \sigma^{2} d \tau+\omega \sigma^{2} d B
$$

where $B$ is a second Brownian motion, and $\alpha$ and $\omega$ are parameters. Here, we have 
omitted $(t+\tau)$ in order to simplify the notation. Hull and White (1987) assume a negative correlation between $W$ and $B$, thereby incorporating leverage effects. The model in (2) is closely related to the GARCH diffusion, which is derived as the diffusion limit of a sequence of GARCH(1,1) models (see Nelson (1990)).

Wiggins (1987) assumes that the log-volatility follows a Gaussian Ornstein-Uhlenbeck (OU) process,

$$
d \log \sigma^{2}=\alpha\left(\mu-\log \sigma^{2}\right) \sigma^{2} d \tau+\omega d B
$$

In the specification, we may introduce leverage effects by assuming a negative correlation between $W$ and $B$. The asymmetric SV model of Harvey and Shephard (1996) is considered to be an Euler-Maruyama approximation of the continuous-time model (3), with negative correlation. Three major extensions of such diffusion-based SV models incorporate jumps to volatility process (Eraker, Johannes and Polson (2003)), model volatility as a function of a number of factors (Chernov et al. (2003)), and allow the log-volatility to follow a long memory process (Comte and Renault (1998)).

If the underlying process of the instantaneous volatility is a continuous-time SV model, the resulting integrated variance is still a stochastic process. At this stage, it may be useful to distinguish the differences and similarities among the conditional variance, stochastic variance, and integrated variance. As shown in Nelson (1990), it is possible to consider the diffusion limits of typical conditional variance models, such as the GARCH model and the exponential GARCH model of Nelson (1991). Hence, conditional variance models are considered to be proxies or approximations of continuous-time SV models. Alternative approximations are the (discrete-time) SV models of Taylor (1982) and Harvey and Shephard (1996), which are obtained by the Euler-Maruyama discretization of the continuous-time SV models. Compared with the class of GARCH models, discrete-time SV models give better approximations in the sense that the latter is straightforwardly derived by the continuous-time SV models. Therefore, the conditional and (discrete-time) stochastic variance can be considered as approximations of the integrated variance obtained by the continuous-time SV models.

There are numerous extensions of GARCH models, and extensions of SV models are still being developed. There are many cases where it is not easy to consider a continuous-time SV model which corresponds to such an extension. For this reason, in the following 
section we consider asymmetric models of the integrated volatility directly.

Although the integrated variance is unobservable, it is possible to estimate it using high frequency data. Such estimates are called “Realized Volatility (RV)”. Zhang, Mykland and Aït-Sahalia (2005) and Barndorff-Nielsen, Hansen, Lunde and Shephard (2007) propose consistent estimator of the integrated variance, under the existence of microstructure noise. For an extensive review of the realized volatility literature, see Bandi and Russell (2006) and McAleer and Medeiros (2008).

\section{Structure of Asymmetric Volatility Models}

In both the conditional and stochastic variance literature, there has been some confusion between the definitions of asymmetry and leverage. This section proposes a new asymmetric volatility model, and examines alternative asymmetric volatility models that have recently been developed and estimated, in order to understand the differences and similarities in alternative definitions of asymmetry and leverage.

\subsection{Asymmetric Volatility Model}

For the return process, we may write

$$
r_{t}=m_{t}+V_{t} z_{t}, \quad z_{t} \sim \text { i.i.d. }(0,1)
$$

where $m_{t}$ and $V_{t}$ are the time-varying mean and volatility processes, and $z_{t}$ is the standardized disturbance. From the definition in the previous section, we have $m_{t}=\int_{0}^{1} \mu(t+\tau-1) d \tau, V_{t}^{2}=\int_{0}^{1} \sigma^{2}(t+\tau-1) d \tau$ and $z_{t} \sim N(0,1)$. Here, we consider a more general model for empirical analysis in a later section. We assume that the log-volatility follows an $\operatorname{ARFIMA}(p, d, q)$ process,

$$
\ln V_{t+1}=\alpha+(1-L)^{-d} \Phi^{-1}(L) \Theta(L) \xi_{t},
$$


where $L$ is the lag-operator, $\Phi(L)$ and $\Theta(L)$ are the lag polynomials for the AR and MA coefficients, and $(1-L)^{d}$ is the fractional difference operator. As discussed in the previous section, the innovation term in the volatility equation plays an important role in considering asymmetry and leverage effects. We suggest a generalized error, such that

$$
\begin{aligned}
& \xi_{t}=\xi_{t}^{*}-E\left(\xi_{t}^{*}\right)+\eta_{t}, \quad \eta_{t} \sim N\left(0, \sigma_{\eta}^{2}\right), \\
& \xi_{t}^{*}=\gamma_{1} z_{t}+\gamma_{2}\left|z_{t}\right|+\gamma_{3} z_{t} I\left(0 \leq z_{t}<\delta\right)+\gamma_{3} \delta I\left(\delta \leq z_{t}\right),
\end{aligned}
$$

where $\gamma_{1}, \gamma_{2}$ and $\gamma_{3}$ are parameters, and $I(0 \leq z<\delta)$ is the indicator function, which

takes the value of one if $0 \leq z<\delta$, and zero otherwise. The first two terms in $\xi_{t}^{*}$ play similar roles as in the EGARCH model. Figure 1 shows the relationship between $\xi$ and $z$, and implies that negative shocks and large positive shocks increase future volatility via $\xi$, but small positive shocks decrease volatility. Such a phenomenon has recently been observed in Chen and Ghysels (2007) by their semi-parametric method using realized volatility.

Consider an AR(1) model of log-volatility, namely, with $d=0, \Phi(L)=1-\phi L$, and $\Theta(L)=1$, such that

$$
\ln V_{t+1}=(1-\phi) \alpha+\phi \ln V_{t}+\xi_{t}
$$

Equation (6) is convenient for comparison with existing SV models:

(i) SV without Leverage: Equations (5) and (6), with restrictions $\gamma_{1}=\gamma_{2}=\gamma_{3}=0$.

(ii) SV with Standardized Leverage: Equations (5) and (6), with restrictions $\gamma_{1}<0$ and $\gamma_{2}=\gamma_{3}=0$. 
(iii) SV with Unstandardized Leverage and Unstandardized Size Effects: Equations (5) and (6), with $\gamma_{3}=0$ and with the replacement of $z_{t}$ by $r_{t}-m_{t}$.

(iv) SV with Standardized Leverage and Unstandardized Size Effects:

Equations (5) and (6), with $\quad \gamma_{3}=0$ and with the replacement of $\left|z_{t}\right|$ by $\left|r_{t}-m_{t}\right|$

(v) SV with Standardized Leverage and Standardized Size Effects: Equations (5) and (6), with $\gamma_{3}=0$.

Model (i) is the basic SV model of Taylor (1982), which is symmetric as positive and negative shocks to returns have identical effects on future volatility. Model (ii) was suggested by Harvey and Shephard (1996), with different notation. Model (iii) was proposed by Danielsson (1994), and was estimated in Asai and McAleer (2005). Model (iv) was suggested by Asai and McAleer (2005) to capture both leverage and asymmetric effects. Model (v) adapts the EGARCH model of Nelson (1991) to the SV literature, and was suggested and estimated by Asai and McAleer (2008). In contrast to Model (iii), Model (v) uses the standardized returns in forecasting future volatility, and can capture various types of asymmetric and leverage effects.

As compared with existing models, the new model in (4) and (5) allows log-volatility to follow the ARFIMA process, and incorporates more flexible asymmetric effects.

\subsection{Types of Asymmetry}

Given the various models presented above, it is useful to consider the following categories of symmetric and asymmetric volatility models, conditional on a negative shock leading to an increase in volatility:

(a) Symmetry: Positive and negative shocks to returns of equal magnitude have identical effects on future volatility;

(b) Asymmetry: Positive and negative shocks to returns of equal magnitude have different effects on future volatility;

(c) Type I Asymmetry (Leverage): A negative correlation exists between current shocks to returns and future volatility; 
(d) Type II Asymmetry: Positive and negative shocks to returns increase future volatility, but a negative shock has a larger effect than does a positive shock of equal magnitude.

(e) Type III Asymmetry: Positive and negative shocks to returns increase future volatility, but a positive shock has a larger effect than does a negative shock of equal magnitude.

(f) Type IV Asymmetry: Negative shocks and large positive shocks increase future volatility, but small positive shocks decrease future volatility. As for Type II Asymmetry, a negative shock has a larger effect on future volatility than does a positive shock of equal magnitude.

Type I Asymmetry, or leverage, is based on the original framework of Black (1976) and Christie (1982), and is also consistent with the definition of leverage for continuous time SV models.

In the conditional volatility literature, the empirical results based on the GJR model of Glosten, Jagannathan and Runkle (1992) and the EGARCH model of Nelson (1991) typically fall into the Type II Asymmetry category. Given the specification, leverage effects are not possible for the GJR model, whereas leverage is possible, though it is frequently not observed, for the EGARCH model.

For the new model in (4) and (5), the basic model arises when $\gamma_{1}=\gamma_{2}=\gamma_{3}=0$. Setting $\gamma_{1}=\gamma_{3}=0$ provides a Symmetric model. Type I Asymmetry is given by $\gamma_{1}<0$, $\left|\gamma_{2}\right|<-\gamma_{1}$ and $\gamma_{3}=0$. Restrictions on the parameters for Type II Asymmetry are given by $\gamma_{1}<0, \gamma_{1}+\gamma_{2}>0$ and $\gamma_{3}=0$. For the case of Type III Asymmetry, the restrictions are $\gamma_{2}<\gamma_{1}<0$ and $\gamma_{3}=0$. Regarding Type IV Asymmetry, the restrictions are $\gamma_{1}<0$, $\gamma_{1}+\gamma_{2}>0, \gamma_{3}<0$ and $\delta>0$.

\section{Model Specification for Realized Volatility}

In this section, we consider the model for realized volatility (RV), based on the discussions in the previous section.

Recently, Asai, McAleer and Medeiros (2008) showed that, even if a bias-corrected and 
consistent estimator of integrated volatility is used, RV will still include measurement errors, called 'Realized Volatility errors', which are not negligible in estimating volatility models.

Let $y_{t}$ be the daily RV, which is a consistent estimate of integrated volatility (IV). The new asymmetric model for RV to be analysed in the paper is given by

$$
\begin{aligned}
& y_{t}=\ln V_{t}+U_{t}, \\
& \ln V_{t+1}=\alpha+(1-L)^{-d} \Phi^{-1}(L) \Theta(L) \xi_{t} \\
& \xi_{t}=\xi_{t}^{*}-E\left(\xi_{t}^{*}\right)+\eta_{t}, \quad \eta_{t} \sim N\left(0, \sigma_{\eta}^{2}\right), \\
& \xi_{t}^{*}=\gamma_{1} z_{t}+\gamma_{2}\left|z_{t}\right|+\gamma_{3} z_{t} I\left(0 \leq z_{t}<\delta\right)-\gamma_{3} \delta I\left(\delta \leq z_{t}\right), \\
& z_{t}=r_{t} / V_{t},
\end{aligned}
$$

where $z_{t}$ is the standardized return and follows the standard normal distribution. This specification enables $U_{t}$ to capture the measurement errors in RV. We will refer to this model as the "RV-ARFIMA $(p, d, q)$-AS $\left(\gamma_{1}, \gamma_{2}, \gamma_{3}\right)$-noise" model. The model allows various types of symmetric and/or asymmetric effects, long-memory property, and takes account of the realized volatility errors. If the measurement errors are neglected, we will have a special case with $\sigma=0$. It should be noted that we consider the mean subtracted return, $r_{t}$, instead of return.

\section{EIS-ML Estimation}

The likelihood function for the asymmetric model in equation (7) includes high-dimensional integration, which is difficult to calculate numerically. We employ the Efficient Importance Sampling (EIS) method developed by Liesenfeld and Richard (2003, 2006) for evaluating the log-likelihood.

The pilot method for the EIS is the Accelerated Gaussian Importance Sampling (AGIS) 
approach, as developed in Danielsson and Richard (1993). The AGIS approach is designed to estimate dynamic latent variable models, where the latent variable follows a linear Gaussian process. While the AGIS technique has limited applicability, the EIS is applicable to models with more flexible classes of distributions and specifications for the latent variables. As in the case of AGIS, EIS is a Monte Carlo technique for the evaluation of high-dimensional integrals. The EIS relies on a sequence of simple low-dimensional least squares regressions to obtain a very accurate global approximation of the integrand. This approximation leads to a Monte Carlo sampler, which produces highly accurate Monte Carlo estimates of the likelihood.

\subsection{Likelihood Evaluation via EIS}

Let $y_{t}$ be an observable variable and $h_{t}=\ln V_{t}$ be a latent variable. We denote the joint density of $Y_{T}=\left\{y_{t}\right\}_{t=1}^{T}$ and $H_{T}=\left\{h_{t}\right\}_{t=1}^{T}$ as $f\left(Y_{T}, H_{T} ; \theta\right)$, indexed by the unknown parameter vector $\theta$. In dynamic latent variable models, the joint density is typically formulated as:

$$
f\left(Y_{T}, H_{T} ; \theta\right)=\prod_{t=1}^{T} f\left(y_{t}, h_{t} \mid Y_{t-1}, H_{t-1}, \theta\right)=\prod_{t=1}^{T} g\left(y_{t} \mid h_{t}, Y_{t-1}, \theta\right) p\left(h_{t} \mid H_{t-1}, Y_{t-1}, \theta\right)
$$

where $g(\square)$ denotes the conditional density of $y_{t}$ given $\left(h_{t}, y_{t-1}\right)$, and $p(\square)$ the conditional density of $h_{t}$ given $\left(H_{t-1}, Y_{t-1}\right)$. For ease of notation, it is assumed that the initial conditions are known constants, but EIS can easily accommodate alternative (stochastic) assumptions.

The likelihood function is given by the $T$-dimensional integral:

$$
L\left(\theta ; Y_{T}\right)=\int f\left(Y_{T}, H_{T} ; \theta\right) d H_{T},
$$

and a natural MC estimate of $L\left(\theta ; Y_{T}\right)$ is given by 


$$
\hat{L}\left(\theta ; Y_{T}\right)=\frac{1}{N} \sum_{i=1}^{N}\left[\prod_{t=1}^{T} g\left(y_{t} \mid \tilde{h}_{t}^{(i)}, Y_{t-1}, \theta\right)\right]
$$

where $\left\{\tilde{h}_{t}^{(i)}(\theta)\right\}_{t=1}^{T}$ denotes a trajectory drawn from the sequence of $T$ densities. Each $\tilde{h}_{t}^{(i)}(\theta)$ is drawn from the conditional density $p\left(h_{t} \mid \tilde{H}_{t-1}^{(i)}(\theta), Y_{t-1}, \theta\right)$.

In order to understand the EIS, we first note that EIS searches for a sequence of samplers that exploits the sample information on $h_{t}$ conveyed by $y_{t}$. Let $\left\{m\left(h_{t} \mid H_{t-1}, x_{t}\right)\right\}_{t=1}^{T}$ denote a sequence of auxiliary samplers, indexed by the auxiliary parameters $X_{n}=\left\{x_{t}\right\}_{t=1}^{T}$. Regardless of the values of the auxiliary parameters, the likelihood function, $L\left(\theta ; Y_{T}\right)$, is rewritten as

$$
L\left(\theta ; Y_{T}\right)=\int \prod_{t=1}^{T}\left[\frac{f\left(y_{t}, h_{t} \mid Y_{t-1}, H_{t-1}, \theta\right)}{m\left(h_{t} \mid H_{t-1}, x_{t}\right)}\right] \prod_{t=1}^{T} m\left(h_{t} \mid H_{t-1}, x_{t}\right) d H_{T}
$$

and the corresponding importance sampling MC estimate of the likelihood is given by

$$
\tilde{L}\left(\theta ; Y_{T}, X_{T}\right)=\frac{1}{N} \sum_{i=1}^{N}\left[\prod_{t=1}^{T}\left\{\frac{f\left(y_{t}, \tilde{h}_{t}^{(i)}\left(x_{t}\right) \mid Y_{t-1}, \tilde{H}_{t-1}^{(i)}\left(x_{t-1}\right), \theta\right)}{m\left(\tilde{h}_{t}^{(i)}\left(x_{t}\right) \mid \tilde{H}_{t-1}^{(i)}\left(x_{t-1}\right), x_{t}\right)}\right\}\right],
$$

where $\left\{\tilde{h}_{t}^{(i)}\left(x_{t}\right)\right\}_{t=1}^{T}$ denotes a trajectory drawn from the sequence of auxiliary importance samplers, $m$.

The EIS chooses a sequence of $m$ densities by selecting values of the auxiliary parameters, $X_{T}$, which provide a good match between the product in the numerator and that in the denominator in equation (9) to minimize the MC sampling variance of $\tilde{L}\left(\theta ; Y_{T}, X_{T}\right)$. In order to implement EIS, it requires constructing a positive functional approximation, 
$k\left(H_{t} ; x_{t}\right)$, for the density $f\left(y_{t}, h_{t} \mid Y_{t-1}, H_{t-1}, \theta\right)$, with the requirement that it be analytically integrable with respect to $h_{t}$. In Bayesian terminology, $k\left(H_{t} ; x_{t}\right)$ plays a role of a density kernel for $m\left(h_{t} \mid H_{t-1}, X_{t}\right)$, which is then given by

$$
m\left(h_{t} \mid H_{t-1}, x_{t}\right)=\frac{k\left(H_{t} ; x_{t}\right)}{\chi\left(H_{t-1}, x_{t}\right)},
$$

where $\chi\left(H_{t-1}, x_{t}\right)=\int k\left(H_{t} ; x_{t}\right) d h_{t}$. Then, the EIS requires solving a back-recursive sequence of low-dimensional least squares problems of the form:

$$
\begin{aligned}
\hat{x}_{t}=\underset{x_{t}}{\arg \min } \sum_{i=1}^{N}\left[\ln \left\{f\left(y_{t}, \tilde{h}_{t}^{(i)}(\theta) \mid Y_{t-1}, \tilde{H}_{t-1}^{(i)}(\theta), \theta\right) \xi\left(\tilde{H}_{t}^{(i)}(\theta), x_{t+1}(\theta)\right)\right\}\right. \\
\left.-c_{t}-\ln k\left(\tilde{H}_{t}^{(i)}(\theta) ; x_{t}\right)\right]^{2}
\end{aligned}
$$

for $t: T \rightarrow 1$, with $\xi\left(H_{T}, x_{T+1}\right) \equiv 1$. As in equation (8), $\left\{\tilde{h}_{t}^{(i)}(\theta)\right\}_{t=1}^{T}$ denotes a trajectory drawn from the $p$ densities, and the $c_{t}$ are unknown constants to be estimated jointly with $x_{t}$. If the density kernel $k\left(H_{t} ; x_{t}\right)$ is chosen within the exponential family of distributions, the EIS least squares problems become linear in $x_{t}$ under the canonical representation of exponential kernels.

The EIS estimate of the likelihood function for a given value of $\theta$ is obtained by substituting $\left\{\hat{x}_{t}(\theta)\right\}_{t=1}^{T}$ for $\left\{x_{t}\right\}_{t=1}^{T}$ in equation (9). In order to obtain maximally efficient importance samplers, a small number of iterations of the EIS algorithm is required, where the natural samplers $p$ are replaced by the previous stage importance samplers. For such iterations to converge to fixed values of the auxiliary parameters, $\hat{x}_{t}$, which are expected to produce optimal importance samplers, it is necessary to apply the technique of Common Random Numbers (CRNs). 


\subsection{Implementation Issues}

As we consider the nonlinear $\operatorname{ARFIMA}(p, d, q)$ process, it is not easy to incorporate it in the likelihood function. Hence, we suggest to employ an $\operatorname{AR}(J)$ approximation of the $\operatorname{AR}(\infty)$ representation of the ARFIMA part. It is similar to the MA $(J)$ approximation of the FIEGARCH model by Bollerslev and Mikkeslen (1996), in the sense that the coefficient of the $J$-th lagged term is almost zero and negligible for large $J$, such as $J=1000$.

Based on the above truncation, we have the distributions of $y_{t}$ and $h_{t}$. The $\operatorname{RV}-\operatorname{ARFIMA}(p, d, q)-\operatorname{AS}\left(\gamma_{1}, \gamma_{2}, \gamma_{3}\right)$-noise model in equation (7) assumes that RVs, $y_{t}$, given the latent log-volatility, $h_{t}$, follows the normal distribution,

$$
g\left(y_{t} \mid h_{t}, \theta\right) \propto \exp \left\{-\frac{1}{2 \sigma^{2}}\left(y_{t}-h_{t}\right)^{2}\right\} .
$$

Conditional on $\left(H_{t-1}, r_{t-1}\right)$, the log-volatility, $h_{t}\left(=\ln V_{t}\right)$, follows the normal distribution,

$$
p\left(h_{t} \mid H_{t-1}, r_{t-1}, \theta\right) \propto \exp \left\{-\frac{1}{2 \sigma_{t}^{2}}\left(h_{t}-\alpha-l_{t}\right)^{2}\right\},
$$

where

$$
l_{t}= \begin{cases}0 & \text { for } t=1 \\ \sum_{i=1}^{t-1} \lambda_{i} h_{t-i}+\xi_{t}^{*}-E\left(\xi_{t}^{*}\right) & \text { for } t=2, \ldots, J \\ \sum_{i=1}^{J} \lambda_{i} h_{t-i}+\xi_{t}^{*}-E\left(\xi_{t}^{*}\right) & \text { for } t=J+1, \ldots, T\end{cases}
$$


and

$$
\sigma_{t}^{2}=\left\{\begin{array}{cr}
\Lambda_{J, J}^{\prime} C_{J} \Lambda_{J, J}+\sigma_{\xi}^{2} & \text { for } t=1 \\
\Lambda_{t, J}^{\prime} C_{J-t+1} \Lambda_{t, J}+\sigma_{\eta}^{2} & \text { for } t=2, \ldots, J \\
\sigma_{\eta}^{2} & \text { for } t=J+1, \ldots, T
\end{array}\right.
$$

with $\xi_{t}^{*}=\gamma_{1} r_{t} e^{-h_{t}}+\gamma_{2}\left|r_{t}\right| e^{-h_{t}}+\gamma_{3} r_{t} e^{-h_{t}} I\left(0 \leq r_{t} e^{-h_{t}}<\delta\right)+\gamma_{3} \delta I\left(\delta \leq r_{t} e^{-h_{t}}\right), \quad \sigma_{\xi}^{2} \quad$ is the variance of $\xi_{t}$ determined by $\left(\gamma_{1}, \gamma_{2}, \gamma_{3}, \delta, \sigma_{\eta}^{2}\right), \Lambda_{t, J}=\left(\lambda_{t}, \lambda_{t+1}, \ldots, \lambda_{J}\right)^{\prime}$ and $C_{j}$ is the unconditional covariance matrix of $\left(h_{1}, \ldots, h_{j}\right)^{\prime}$.

We chose $m$ as the parametric extensions of the natural samplers, $p$. Hence, the parameterization for $k$ is given by

$$
k\left(H_{t} ; x_{t}, r_{t-1}\right)=p\left(h_{t} \mid H_{t-1}, r_{t-1}, \theta\right) \zeta\left(h_{t}, x_{t}\right) \text {, }
$$

where the auxiliary function $\zeta\left(h_{t}, x_{t}\right)$ is itself a Gaussian density kernel. Under this parameterization, the natural sampler, $p$, cancels out in the least squares problem in equation (11), to the effect that $\ln \zeta\left(h_{t}, x_{t}\right)$ serves to approximate $\ln g\left(y_{t} \mid h_{t}, Y_{t-1}, r_{t-1}, \theta\right)$ $+\ln \chi\left(H_{t}, x_{t+1}, r_{t}\right)$. In particular, the appropriate auxiliary function for the asymmetric model is given by $\ln \zeta\left(h_{t}, x_{t}\right)=\exp \left(x_{1 t} h_{t}+x_{2 t} h_{t}\right)$, with $x_{t}=\left(x_{1 t}, x_{2 t}\right)$, and the density kernels of the importance samplers have the form

$$
k\left(H_{t} ; x_{t}, r_{t-1}\right) \propto \exp \left[-\frac{1}{2}\left\{\left(\frac{\lambda_{t}}{\sigma_{t}}\right)^{2}-2\left(\frac{\alpha+l_{t}}{\sigma_{t}^{2}}+x_{1 t}\right) h_{t}+\left(\frac{1}{\sigma_{t}^{2}}-2 x_{2 t}\right) h_{t}^{2}\right\}\right] .
$$

Accordingly, the conditional mean and variance of $h_{t}$ on $m$ are given by 


$$
\mu_{m, t}=\sigma_{m, t}^{2}\left(\frac{\alpha+l_{t}}{\sigma_{t}^{2}}+x_{1 t}\right), \quad \sigma_{m, t}^{2}=\frac{\sigma_{t}^{2}}{1-2 \sigma_{t}^{2} x_{2 t}}
$$

respectively. Integrating $k\left(H_{t} ; x_{t}, r_{t-1}\right)$ with respect to $h_{t}$, and omitting irrelevant multiplicative factors, leads to the following expression for the integrating constant:

$$
\chi\left(H_{t-1}, x_{t}, r_{t-1}\right) \propto \exp \left\{\frac{\mu_{m, t}^{2}}{2 \sigma_{m, t}^{2}}-\frac{\left(\alpha+l_{t}\right)^{2}}{2 \sigma_{t}^{2}}\right\} .
$$

Based upon these functional forms, the computation of an EIS estimate of the likelihood for the asymmetric model requires the following steps:

Step (0): Use the natural samplers, $p$, to draw $N$ trajectories of the latent variable, $\left\{\tilde{h}_{t}^{(i)}(\theta)\right\}_{t=1}^{T}$

Step $(t):(t: T \rightarrow 1):$ Use these random draws to solve the back-recursive sequence of least squares problems, as defined in equation (11). The step $t$ least squares problem is characterized by the following linear auxiliary regression:

$$
\begin{aligned}
- & \frac{1}{2 \sigma^{2}}\left(y_{t}-h_{t}\right)^{2}+\ln \chi\left(\tilde{h}_{t}^{(i)}(\theta), \hat{x}_{t+1}(\theta)\right) \\
& =\text { constant }+x_{1 t} \tilde{h}_{t}^{(i)}(\theta)+x_{2 t}\left\{\tilde{h}_{t}^{(i)}(\theta)\right\}^{2}+u_{t}^{(i)}, \quad i: 1 \rightarrow N,
\end{aligned}
$$

where $u_{t}^{(i)}$ denotes the regression error term. The initial condition for the integrating constant (in equation (13)) is given by $\chi\left(h_{T}, x_{T+1}, r_{T}\right) \equiv 1$. 
Step $(\mathbf{T}+\mathbf{1})$ : The EIS samplers, $\left\{m\left(h_{t} \mid H_{t-1}, \hat{x}_{t}(\theta)\right)\right\}_{t=1}^{T}$, which are characterized by the conditional mean and variance given in equation (12), are used to draw $N$ trajectories $\left\{\tilde{h}_{t}^{(i)}\left(\hat{a}_{t}(\theta)\right)\right\}_{t=1}^{T}$, from which the EIS estimate of the likelihood is calculated according to equation (9).

We set $N=50$, as Liesenfeld and Richard (2003) reported that 50 is sufficient for univariate and nonlinear latent variable models, such as SV. After 7-10 iterations, $\tilde{L}\left(\theta ; Y_{T}, X_{T}, R_{T}\right)$ converged for each $\theta$. The next section gives the EIS-ML estimates for the asymmetric model of RV.

For the case of neglecting measurement errors (that is, $\sigma=0$ ), $h_{t}$ is observable, so it is possible to perform maximum likelihood estimation without simulations. By comparing the log-likelihood with the EIS log-likelihood above, we have the conventional likelihood ratio test statistics, which follows the $\chi^{2}(1)$ distribution under the null hypothesis that $\sigma=0$

\section{Empirical Results}

The empirical analysis focuses on the RV of Standard and Poor's 500 Composite Index. In order to estimate the daily realized volatility, we use the two time scales estimator (TTSE) of Zhang, Mykland and Aït-Sahalia (2005) with five-minute grids, which is a consistent estimator of the daily realized volatility. The sample period is Jan/3/1996 to March/29/2007, giving $T=2796$ observations of RV.

As a preliminary analysis, we consider the new Fractional Integrated EGARCH- $t$ models given in Section 3 as 


$$
\begin{aligned}
& r_{t}=\sigma_{t} z_{t}, \quad z_{t} \sim S t(v), \\
& \ln \sigma_{t+1}^{2}=\alpha+(1-L)^{-d} \Phi^{-1}(L) \Theta(L) \xi_{t}, \\
& \xi_{t}=\xi_{t}^{*}-E\left(\xi_{t}^{*}\right), \\
& \xi_{t}^{*}=\gamma_{1} z_{t}+\gamma_{2}\left|z_{t}\right|+\gamma_{3} z_{t} I\left(0 \leq z_{t}<\delta\right)-\gamma_{3} \delta I\left(\delta \leq z_{t}\right),
\end{aligned}
$$

where $S t(v)$ denotes the standardized $t$ distribution, with degrees of freedom given by $v$. Note that this model implicitly specifies that $\sigma_{\eta}=0$, so that $\sigma_{t}$ is determined by the past information. We denote the above model as the FIEGARCH $(p, d, q)-t-\mathrm{AS}\left(\gamma_{1}, \gamma_{2}, \gamma_{3}\right)$ model and, for the case where $d=0$, as the $\operatorname{EGARCH}(p, q)-t$-AS $\left(\gamma_{1}, \gamma_{2}, \gamma_{3}\right)$ model.

We estimated two kinds of models, EGARCH(1,1)-t-AS $\left(\gamma_{1}, \gamma_{2}, \gamma_{3}\right)$ and FIEGARCH(1,d,1)-t-AS $\left(\gamma_{1}, \gamma_{2}, 0\right)$. Table 1 shows the ML estimates of these models. For the former model, all the estimated parameters, except for $\alpha$ and $\gamma_{3}$, are significant at the five percent level. The estimate of $\phi$ is close to 0.99 , showing high persistence in volatility. The estimate of $\gamma_{1}$ is negative, while that of $\gamma_{2}$ is positive. The estimate of $1 / v$ is 0.11 , indicating that the estimate of $v$ is close to 9 . The results are typical for the EGARCH- $t$ specification. For the long memory model, all the estimated parameters, except for $\gamma_{1}$ and $1 / v$, are significant. This specification shows the lack of importance of asymmetric effects and heavy-tailed conditional distributions. Similar results are also found in the literature with the FIEGARCH-t specification. Regarding the type of asymmetry, the estimates of the former model indicate Type II asymmetry, while those of the latter model display Symmetry.

In the following, we will show that the empirical results are completely different in the case of RV. It should be noted that it is inadequate to compare the log-likelihood of the EGARCH models with that of RV models as the former is based on $r_{t}$ while the latter is 
based on the RV, $y_{t}$. Furthermore, the fat tails of the conditional distribution of $r_{t}$ are irrelevant for the RV model.

Table 2 shows the EIS-ML results of the RV-AR(1)-AS $\left(\gamma_{1}, \gamma_{2}, \gamma_{3}\right)$-noise model. Regarding asymmetry, we consider four specifications, namely $\operatorname{AS}(0,0,0), \operatorname{AS}\left(\gamma_{1}, 0,0\right)$, $\operatorname{AS}\left(\gamma_{1}, \gamma_{2}, 0\right)$, and $\operatorname{AS}\left(\gamma_{1}, \gamma_{2}, \gamma_{3}\right)$. All the estimated parameters are significant at the five percent level. The the AS $\left(\gamma_{1}, \gamma_{2}, \gamma_{3}\right)$ model has the smallest AIC and BIC, we report the results only for this specification.

The estimate of $\sigma$ is close to 0.4 , showing that the RV errors are not negligible. The estimate of $\phi$ is 0.986 , while that of $\sigma_{\eta}$ is 0.11 , which are typical of SV models. The estimate of $\gamma_{1}$ is negative, while that of $\gamma_{2}$ is positive. Unlike the estimates of the EGARCH model, the estimate of $\gamma_{3}$ is negative and significant. Figure 2 gives the news impact from $z_{t}$ to $\ln V_{t+1}$, showing that negative shocks and large positive shocks increase future volatility, but small positive shocks decrease volatility. In other words, Figure 2 displays Type IV Asymmetry.

Table 3 presents the EIS-ML results for the RV-ARFIMA(1,d,0)-AS $\left(\gamma_{1}, \gamma_{2}, \gamma_{3}\right)$-noise model. As before, we consider four kinds of asymmetric effects. The AIC and BIC selected the AS $\left(\gamma_{1}, \gamma_{2}, \gamma_{3}\right)$ model, so we will concentrate the analysis on this model. All the estimated parameters are significant at the five percent level. The estimate of $\sigma$ is close to 0.4 , indicating that the RV errors are not negligible. The estimate of $d$ is 0.47 , showing that the log-volatility has long memory and is a stationary process. The estimate of $\phi$ is positive and close to 0.4 , which is against the typical value of -0.1 in the RV literature. The difference can be explained by the existence of RV errors, $U_{t}=y_{t}-\ln V_{t}$. As shown in the Monte Carlo experiments of Asai, McAleer and Medeiros (2008), even minor RV errors cause bias in the estimates if the RV error is neglected in estimation. The signs of $\gamma_{1}, \gamma_{2}$ and $\gamma_{3}$ are the same as in the case of Table 2. Figure 3 shows the news impact from $z_{t}$ to $\ln V_{t+1}$, and the curve is classified as Type IV asymmetry. 
From Tables 2 and 3, we find that the RV-ARFIMA(1,d,0)-AS $\left(\gamma_{1}, \gamma_{2}, \gamma_{3}\right)$-noise model has the smallest AIC, while BIC chooses the RV-AR(1)-AS $\left(\gamma_{1}, \gamma_{2}, \gamma_{3}\right)$-noise model. These tables indicate that including the additional term, $\gamma_{3}$, significantly improves the goodness of fit of the model.

\section{Concluding Remarks}

In both the conditional and stochastic volatility literature, there has been some confusion between the definitions of asymmetry and leverage. In order to clarify this issue, the paper showed the relationship among conditional, stochastic, integrated and realized volatility. Then we proposed a new asymmetric volatility model, which sensitively capture the effects of large and small, and positive and negative, shocks. Based on the new specification, this paper examined alternative univariate volatility models that have recently been developed and estimated in order to understand the differences and similarities in the definitions of asymmetry and leverage.

We extended the specification of asymmetric volatility in order to model RV by taking account of the RV errors. This is a general model which includes not only various kinds of asymmetric effects, but also short and long memory specifications. We applied the EIS-ML method to estimate the model of RV.

The empirical results for the RV of Standard and Poor's 500 Composite Index showed the existence of RV errors. The estimates of the short and long memory models supported the Type IV asymmetry definition, which satisfies the following three conditions: (i) negative shocks to returns increase future volatility; (ii) large positive shocks to returns increase future volatility, but a negative shock has a larger effect on volatility than does a positive shock of equal magnitude; and (iii) small positive shocks to returns decrease future volatility. 


\section{References}

Asai, M. and M. McAleer (2005), "Dynamic Asymmetric Leverage in Stochastic Volatility Models”, Econometric Reviews, 24, 317-332.

Asai, M. and M. McAleer (2008), “Alternative Asymmetric Stochastic Volatility Models”, to appear in Econometric Reviews.

Asai, M., M. McAleer and M. Medeiros (2008), "Modelling and Forecasting Daily Volatility with Noisy Realized Volatility Measures”, Unpublished paper, Soka University.

Asai, M., M. McAleer and J. Yu (2006), "Multivariate Stochastic Volatility: A Review”, Econometric Reviews, 25, 145-175.

Bandi, F.M. and J.R. Russell (2006), "Market Microstructure Noise, Integrated Variance Estimators, and the Accuracy of Asymptotic Approximations”, unpublished paper, Graduate School of Business, University of Chicago.

Barndorff-Nielsen, O.E., P.H. Hansen, A. Lunde and N. Shephard (2007), "Subsampling Realised Kernels”, unpublished manuscript, Stanford University.

Black, F. (1976), “Studies of Stock Market Volatility Changes”, 1976 Proceedings of the American Statistical Association, Business and Economic Statistics Section, pp. 177-181.

Bollerslev, T. and H.O. Mikkeslen (1996), "Modeling and Pricing Long Memory in Stock Market Volatility”, Journal of Econometrics, 73, 151-184.

Chen, X. and E. Ghysels (2007), "News - Good or Bad - and its Impact Over Multiple Horizons”, Unpublished paper, University of North Carolina at Chapel Hill.

Chernov, M., A.R. Gallant, E. Ghysels and G. Tauchen (2003), “Alternative Models for Stock Price Dynamics”, Journal of Econometrics, 116, 225 - 257.

Christie, A.A. (1982), “The Stochastic Behavior of Common Stock Variances: Value, Leverage and Interest Rate Effects”, Journal of Financial Economics, 10, 407-432. 
Comte, F. and E. Renault (1998), "Long Memory in Continuous-Time Stochastic Volatility Models”, Mathematical Finance, 8, 291-323.

Danielsson, J. (1994), "Stochastic Volatility in Asset Prices: Estimation with Simulated Maximum Likelihood”, Journal of Econometrics, 64, 375-400.

Danielsson, J., and J.-F. Richard (1993), "Quadratic Acceleration for Simulated Maximum Likelihood Evaluation”, Journal of Applied Econometrics, 8, 153-173.

Durbin, J., and S.J. Koopman (1997), “Monte Carlo Maximum Likelihood Estimation for Non-Gaussian State Space Models”, Biometrika, 84, 669-684.

Eraker, B., M. Johannes, and N.G. Polson (2003), “The Impact of Jumps in Returns and Volatility”, Journal of Finance, 53, 1269-1300.

Ghysels, E., A.C. Harvey and E. Renault (1996), “Stochastic Volatility”, In C. R.Rao and G. S. Maddala eds., Statistical Methods in Finance, pp. 119-191. Amsterdam: North-Holland.

Glosten, L., R. Jagannathan and D. Runkle (1992), "On the Relation Between the Expected Value and Volatility of Nominal Excess Returns on Stocks”, Journal of Finance, 46, 1779-1801.

Hansen, P.R., J. Large and A. Lunde (2008), "Moving Average-based Estimators of Integrated Variance”, Econometric Reviews, 27, 79-111.

Harvey, A.C. and N. Shephard (1996), "Estimation of an Asymmetric Stochastic Volatility Model for Asset Returns”, Journal of Business and Economic Statistics, 14, 429-434.

Hull, J. and A. White (1987), "The Pricing of Options on Assets with Stochastic Volatility”, Journal of Finance, 42, 281-300.

Liesenfeld, R., and J.-F. Richard (2003), "Univariate and Multivariate Stochastic Volatility Models: Estimation and Diagnostics”, Journal of Empirical Finance, 10, 
505-531.

Liesenfeld, R., and J.-F. Richard (2006), “Classical and Bayesian Analysis of Univariate and Multivariate Stochastic Volatility Models”, Econometric Reviews, 25, 335-361.

McAleer, M. (2005), "Automated Inference and Learning in Modeling Financial Volatility”, Econometric Theory, 21, 232-261.

McAleer, M. and M. Medeiros (2008), “Realized Volatility: A Review”, Econometric Reviews, 27, 10-45.

Nelson, D.B. (1990), “ARCH Models as Diffusion Approximations”, Journal of Econometrics, 45, 7-38.

Nelson, D.B. (1991), “Conditional Heteroskedasticity in Asset Returns: A New Approach”, Econometrica, 59, 347-370.

Taylor, S.J. (1982), "Financial Returns Modelled by the Product of Two Stochastic Processes - A Study of Daily Sugar Prices 1961-79”, In O. D. Anderson (Ed.), Time Series Analysis: Theory and Practice, 1, pp. 203-226, Amsterdam: North-Holland.

Wiggins, J.B. (1987), “Option Values Under Stochastic Volatility: Theory and Empirical Estimates”, Journal of Financial Economics, 19, 351-372.

Zhang, L., P.A. Mykland and Y. Aït-Sahalia (2005), “A Tale of Two Time Scales: Determining Integrated Volatility with Noisy High Frequency Data”, Journal of the American Statistical Association, 100, 1394 - 1411. 
Table 1: ML Estimates of New EGARCH Class

\begin{tabular}{c|cc|cc}
\hline Parameters & \multicolumn{2}{|c|}{ New EGARCH- $t$} & \multicolumn{2}{|c}{ FIEGARCH(1,d,0)- $t$} \\
\hline$d$ & & & 0.4067 & $(0.0256)$ \\
$\phi$ & 0.9856 & $(0.0034)$ & -0.2651 & $(0.0450)$ \\
$\alpha$ & 0.1937 & $(0.2013)$ & 0.3101 & $(0.0777)$ \\
$\gamma_{1}$ & -0.1004 & $(0.0122)$ & 0.0115 & $(0.0441)$ \\
$\gamma_{2}$ & 0.1025 & $(0.0142)$ & 2.4407 & $(0.0776)$ \\
$\gamma_{3}$ & -0.0295 & $(0.0211)$ & & \\
$\delta$ & 0.5049 & $(0.0438)$ & & \\
$1 / v$ & 0.1109 & $(0.0165)$ & 0.0004 & $(0.0410)$ \\
Log-Like & -3872.87 & & -- & \\
AIC & 7759.74 & & -- & \\
BIC & 7801.30 & & -- & \\
\hline
\end{tabular}

Note: Standard errors are given in parentheses. With respect to FIEGARCH, the log-likelihood and other statistics are not reported, as they are not comparable because of 1000 initial values. 
Table 2: EIS Estimates of RV-AR(1)-AS $\left(\gamma_{1}, \gamma_{2}, \gamma_{3}\right)$-noise

\begin{tabular}{|c|c|c|c|c|}
\hline Parameters & $\operatorname{AS}(0,0,0)$ & $\operatorname{AS}\left(\gamma_{1}, 0,0\right)$ & $\operatorname{AS}\left(\gamma_{1}, \gamma_{2}, 0\right)$ & $\operatorname{AS}\left(\gamma_{1}, \gamma_{2}, \gamma_{3}\right)$ \\
\hline$\phi$ & $\begin{array}{c}0.9747 \\
(0.0051)\end{array}$ & $\begin{array}{c}0.9728 \\
(0.0040)\end{array}$ & $\begin{array}{c}0.9870 \\
(0.0044)\end{array}$ & $\begin{array}{c}0.9856 \\
(0.0044)\end{array}$ \\
\hline$\sigma_{\eta}$ & $\begin{array}{c}0.1478 \\
(0.0091)\end{array}$ & $\begin{array}{c}0.1111 \\
(0.0075)\end{array}$ & $\begin{array}{c}0.1110 \\
(0.0074)\end{array}$ & $\begin{array}{c}0.1103 \\
(0.00720)\end{array}$ \\
\hline$\alpha$ & $\begin{array}{l}-0.3148 \\
(0.1091)\end{array}$ & $\begin{array}{l}-0.1795 \\
(0.0788)\end{array}$ & $\begin{array}{l}-0.8439 \\
(0.3227)\end{array}$ & $\begin{array}{c}1.2246 \\
(0.5127)\end{array}$ \\
\hline$\gamma_{1}$ & & $\begin{array}{l}-0.0681 \\
(0.0046)\end{array}$ & $\begin{array}{l}-0.0649 \\
(0.0043)\end{array}$ & $\begin{array}{l}-0.0418 \\
(0.0062)\end{array}$ \\
\hline$\gamma_{2}$ & & & $\begin{array}{c}0.0424 \\
(0.0074)\end{array}$ & $\begin{array}{c}0.0561 \\
(0.0079)\end{array}$ \\
\hline$\gamma_{3}$ & & & & $\begin{array}{c}-0.1934 \\
(0.0471)\end{array}$ \\
\hline$\delta$ & & & & $\begin{array}{c}0.4902 \\
(0.0605)\end{array}$ \\
\hline$\sigma$ & $\begin{array}{c}0.4054 \\
(0.0073)\end{array}$ & $\begin{array}{c}0.4092 \\
(0.0067)\end{array}$ & $\begin{array}{c}0.4125 \\
(0.0067)\end{array}$ & $\begin{array}{c}0.4116 \\
(0.0067)\end{array}$ \\
\hline Log-Like & -1921.94 & -1821.52 & -1806.24 & -1793.51 \\
\hline AIC & 3851.88 & 3653.04 & 3624.48 & 3603.03 \\
\hline BIC & 3875.63 & 3682.72 & 3660.10 & 3650.52 \\
\hline
\end{tabular}

Note: Standard errors are in parentheses. 
Table 3: EIS Estimates of RV-ARFIMA(1,d,0)-AS $\left(\gamma_{1}, \gamma_{2}, \gamma_{3}\right)$-noise

\begin{tabular}{|c|c|c|c|c|}
\hline Parameters & $\operatorname{AS}(0,0,0)$ & $\operatorname{AS}\left(\gamma_{1}, 0,0\right)$ & $\operatorname{AS}\left(\gamma_{1}, \gamma_{2}, 0\right)$ & $\operatorname{AS}\left(\gamma_{1}, \gamma_{2}, \gamma_{3}\right)$ \\
\hline$d$ & $\begin{array}{c}0.4955 \\
(0.0039)\end{array}$ & $\begin{array}{c}0.4987 \\
(0.00089)\end{array}$ & $\begin{array}{c}0.4748 \\
(0.0090)\end{array}$ & $\begin{array}{c}0.4727 \\
(0.0076)\end{array}$ \\
\hline$\phi$ & $\begin{array}{c}0.3261 \\
(0.0603)\end{array}$ & $\begin{array}{c}0.3676 \\
(0.0438)\end{array}$ & $\begin{array}{c}0.4166 \\
(0.0538)\end{array}$ & $\begin{array}{c}0.4373 \\
(0.0291)\end{array}$ \\
\hline$\sigma_{\eta}$ & $\begin{array}{c}0.2416 \\
(0.0225)\end{array}$ & $\begin{array}{c}0.1750 \\
(0.0147)\end{array}$ & $\begin{array}{c}0.1852 \\
(0.0157)\end{array}$ & $\begin{array}{c}0.1739 \\
(0.0080)\end{array}$ \\
\hline$\alpha$ & $\begin{array}{c}-0.5832 \\
(0.2394)\end{array}$ & $\begin{array}{c}0.0021 \\
(0.0026)\end{array}$ & $\begin{array}{c}0.0051 \\
(0.0027)\end{array}$ & $\begin{array}{c}0.0046 \\
(0.0020)\end{array}$ \\
\hline$\gamma_{1}$ & & $\begin{array}{l}-0.0865 \\
(0.0061)\end{array}$ & $\begin{array}{l}-0.0827 \\
(0.0063)\end{array}$ & $\begin{array}{l}-0.0275 \\
(0.0075)\end{array}$ \\
\hline$\gamma_{2}$ & & & $\begin{array}{c}0.0226 \\
(0.0077)\end{array}$ & $\begin{array}{c}0.0511 \\
(0.0076)\end{array}$ \\
\hline$\gamma_{3}$ & & & & $\begin{array}{l}-0.2428 \\
(0.0287)\end{array}$ \\
\hline$\delta$ & & & & $\begin{array}{c}0.8841 \\
(0.0196)\end{array}$ \\
\hline$\sigma$ & $\begin{array}{c}0.3648 \\
(0.01208)\end{array}$ & $\begin{array}{c}0.3844 \\
(0.0081)\end{array}$ & $\begin{array}{c}0.3827 \\
(0.0085)\end{array}$ & $\begin{array}{c}0.3858 \\
(0.0067)\end{array}$ \\
\hline Log-Like & -1908.27 & -1819.30 & -1811.37 & -1792.16 \\
\hline AIC & 3826.54 & 3650.60 & 3636.75 & 3602.31 \\
\hline BIC & 3856.22 & 3686.22 & 3678.30 & 3655.74 \\
\hline
\end{tabular}

Note: Standard errors are in parentheses. 

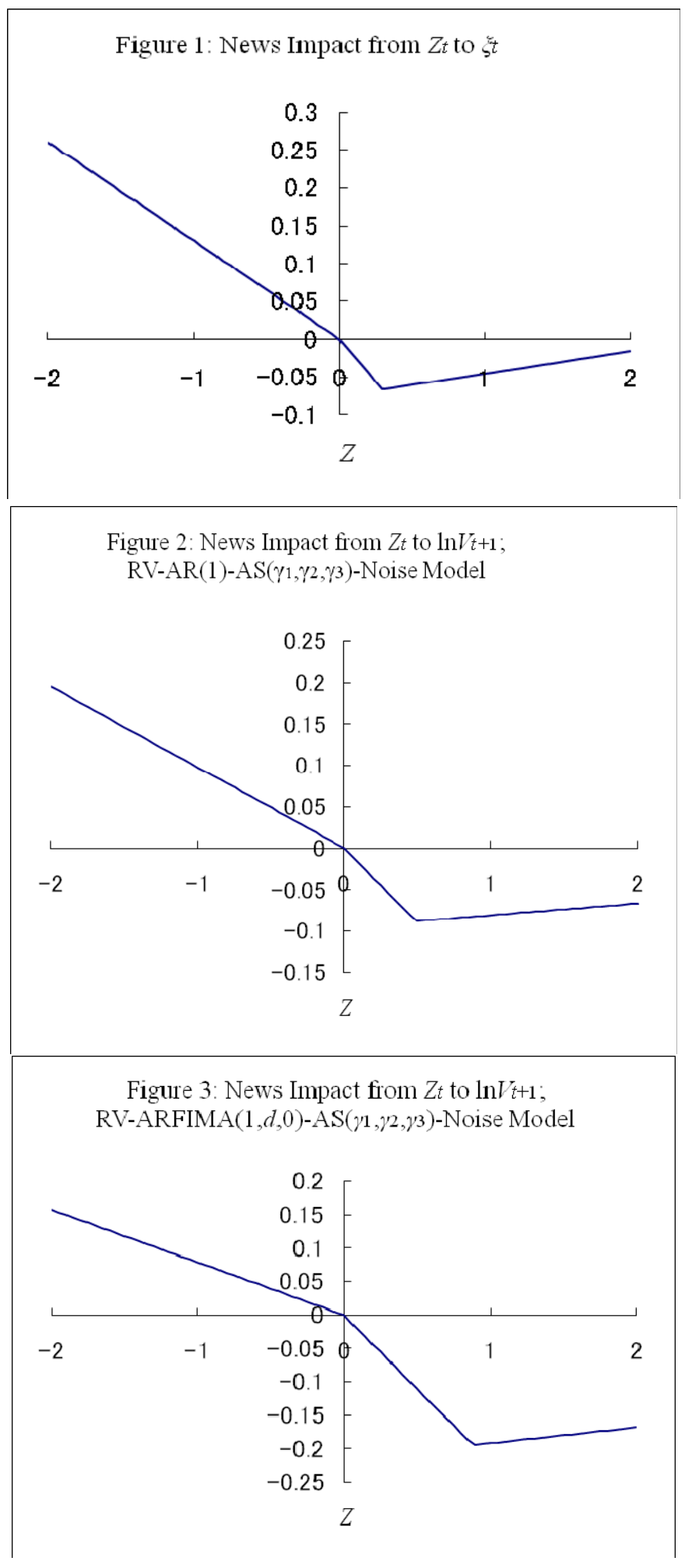